\title{
$A b$ initio studies of staggered Li adatoms on graphene
}

\author{
R.E. Mapasha, N. Chetty* \\ Physics Department, University of Pretoria, Pretoria 0001, South Africa
}

\section{A R T I C L E I N F O}

\section{Article history:}

Received 31 March 2010

Received in revised form 8 June 2010

Accepted 10 June 2010

Available online xxxx

\section{Keywords:}

Graphene

Adatoms of $\mathrm{Li}$

Electronic structure

Density functional theory within the

generalized gradient approximation

\begin{abstract}
A B S T R A C T
We study Li on graphene using the Vienna $a b$ initio Simulation Package employing the projector-augmented wave method within the generalized gradient approximation for the exchange-correlation potential. We give detailed structural and electronic results for various configurations involving Li on the $(1 \times 1),(2 \times 1)$ and $(2 \times 2)$ two-dimensional unit cells, and we consider the isolated Li dimer on graphene. We consider more detailed configurations than have been studied before, and our results compare favourably with previously calculated results where such results exist. For $100 \%$ coverage, we have new results for Li on the on-top site, which suggests a staggered configuration for the lowest energy structure for which the Li adatoms are alternately pushed into and pulled out of the graphene layer. For $50 \%$ coverage, Li favours the hollow site. We have discovered that a careful relaxation of the system also shows a staggered configuration, a result that has not been investigated before.
\end{abstract}

(c) 2010 Elsevier B.V. All rights reserved.

\section{Introduction}

Carbon exhibits a variety of interesting structural and electronic systems because of its propensity to form both $s p^{2}$ and $s p^{3}$ bonds. This gives rise to a range of interesting magnetic, transport and physical properties for carbon and carbon-based materials - especially nanoscaled materials - with a wide range of both established and potentially new applications. This contrasts strongly with silicon, for example, which in principle should be very similar since it is only one element below carbon in the periodic table. Silicon does not form into the multitude of structural systems that carbon does, such as nanotubes, graphite, buckeyballs and, more latterly, graphene.

The advent of graphene by Novoselov et al. [1] in 2004 has excited much interest in these unique two-dimensional systems because of the enormous potential that graphene has for novel electronic, magnetic and optical applications [2]. These systems also provide theoreticians with the opportunity to explore novel methods for modelling these systems because of their ideal twodimensional nature. Graphene, which may be considered to be a single layer of graphite, is a semi-metal with a unique electronic structure [3]. The low energy electronic excitations are massless because of the infinite curvature of the occupied bands at the point-like Fermi surface which results in ballistic transport [4-6].

A great effort recently has been centred on altering the electronic structure of graphene using various dopants. Hydrogen is the most obvious choice of dopant, and hydrogenated graphene, which is more commonly referred to as graphane [7,8], has been

\footnotetext{
* Corresponding author. Tel.: +27214206204.

E-mail address: Nithaya.Chetty@up.ac.za (N. Chetty).
}

studied very extensively [9]. From previous work, the main results for our purposes are two fold: firstly, the energetically most favoured site for a single isolated hydrogen adatom on graphene is the on-top site and, secondly, $100 \%$ coverage at the on-top sites results in insulating behaviour, with the lowest energy configuration corresponding to hydrogen adatoms attaching alternately above and below the graphene layer.

Simple electron counting arguments suggest that all the dangling bond states are saturated in this case which results in insulating behaviour. Electronic structure calculations of this system yield a relatively large band-gap in the region of $3.5 \mathrm{eV}$ [10] for this material.

Li on graphene has also been studied by several previous authors [11-14], and here we extend this study to newer configurations, and we give more detailed structural and electronic results. In our computations, we consider Li on the on-top site. For $100 \%$ coverage for $\mathrm{Li}$ on the on-top sites, we find results that are opposite to those of hydrogen on graphene: we find that Li attaching on the same side of the graphene sheet is lower in energy compared with Li attaching alternately on opposite sides of the graphene sheet. We have calculated the detailed staggering of the Li adatoms in this case. An important difference between $\mathrm{H}$ on graphene compared with $\mathrm{Li}$ on graphene is that $\mathrm{H}$ forms covalent bonds with $\mathrm{C}$, whereas our work shows that Li forms ionic bonds with $\mathrm{C}$.

The most favoured site for a single isolated Li adatom on graphene is the hollow site. This means that the maximum coverage that is attainable for Li at the hollow sites is only 50\%. This results in an under-saturation of the dangling bond states and this gives rise to metallic behaviour. Our detailed relaxations show that the Li adatoms are staggered on the graphene layer. 
We explore Li molecular structures on graphene in this paper using first principles total energy methods applied to the $(1 \times 1)$, $(2 \times 1),(2 \times 2)$ and the $(5 \times 5)$ two-dimensional cells. For different coverages we have different ground state configurations. Our work shows that for $100 \%$ coverage on the on-top site the ground state corresponds to Li attaching on the same side in a staggered configuration. For $50 \%$ coverage, we have shown that the hollow site is favoured over the on-top site. The ground state configuration for this coverage corresponds to $\mathrm{Li}$ attaching on the same side in a staggered configuration. For even lower coverages, the hollow site is deemed to be more favourable. However, for very low coverages, the attachment on the same side or on opposite sides of the graphene sheet becomes degenerate.

In the next section, we briefly outline our computational methodology. In Section 3, we consider the various different configurations involving Li on graphene. We draw our conclusions in Section 4.

\section{Methodology}

Our computational method is based on density functional theory where we used the Vienna ab initio Simulation Package (VASP) [1518] within the generalized gradient approximation [19] for the exchange-correlation potential. The VASP implementation is based on the projector-augmented wave method (PAW) [20]. We included the semi-core $1 s$ states of Li explicitly as valence electrons in the construction of the PAW. Our calculations were done at an energy cutoff $500 \mathrm{eV}$ for the plane wave expansion set for the electron wavefunctions. The total energy is converged to within $10^{-7} \mathrm{eV}$.

We have performed both non-magnetic and spin-polarised calculations for all of the $(1 \times 1)$ cell systems, and we conclude that the non-magnetic choice is sufficiently accurate. In many instances, there are only slight changes to the bands above the Fermi level with the choice of spin-polarisation, where there are negligible effects on the total energies and bond lengths for these systems. All subsequent calculations were done treating the system as being non-magnetic.

The Monkhorst-Pack [21] scheme was used for the sampling of the Brillouin zone. We tested the convergence of our results for energy differences and structural parameters applied to the $(1 \times 1)$ conventional two-dimensional cell and we concluded that the $(10 \times 10 \times 1)$ sampling of the Brillouin is sufficiently converged. For all subsequent calculations, we use a Monkhorst-Pack grid of comparable density to that for the $(10 \times 10 \times 1)$ grid applied to the $(1 \times 1)$ conventional cell.

We use a periodic supercell geometry that exploits the plane wave formulation of the electronic structure problem. The separation for the graphene layers in the supercell was set at $15 \AA$, to avoid spurious interlayer interactions. Our calculated lattice parameter for the two-dimensional hexagonal primitive unit cell is $2.47 \AA$, which is comparable to the experimental value of $2.46 \AA$ [1] and other published theoretical results of 2.465 [22].

The electronic states were occupied in accordance with the Fermi distribution function, with a Fermi smearing parameter of $k_{B} T=0.2 \mathrm{eV}$. The atomic forces were calculated using the Hellman-Feynman theorem, and the atomic positions were relaxed until the forces were reduced to less than $0.01 \mathrm{eV} / \AA$.

The energy of a single isolated $\mathrm{Li}$ atom was computed in a box of size $(10 \times 11 \times 12) \AA^{3}$ using the above methodology but for $\Gamma$ only sampling of the Brillouin zone and using spin-polarisation for the exchange-correlation effects.

\section{Li adatoms on graphene}

Chan et al. studied the equilibrium properties of a single isolated $\mathrm{Li}$ adatom on graphene [11]. They considered a $(4 \times 4)$ con- ventional cell, which they argued was sufficiently large to minimize adatom-adatom interactions. They found that the hollow site, with a binding energy of $1.096 \mathrm{eV}$, was energetically most favourable, followed by the bridge site, with a binding energy $0.773 \mathrm{eV}$, and followed by the on-top site with a binding energy of $0.754 \mathrm{eV}$. Furthermore, Chan et al. concluded that the binding was ionic in nature with charge transferred from the Li to the graphene substrate with no significant change to the occupied graphene bands.

\subsection{The $(1 \times 1)$ cell}

For $50 \%$ coverage, we compare a single Li adatom at the on-top site with the hollow site for a $(1 \times 1)$ cell of graphene. Our results for the fully relaxed systems indicate that the hollow site is lower in energy compared to the on-top site by only $0.046 \mathrm{eV}$. This is a relatively small energy difference, which shows that under conditions of finite temperature, islands of hollow sites may co-exist with islands of alternate on-top sites. Not surprisingly, these systems are metallic in nature as can be deduced by simple electron counting arguments. The $\mathrm{Li}-\mathrm{C}$ bond length for the on-top site is $2.19 \AA$ and for the hollow site is $1.85 \AA$. The shorter bond length at the hollow site is understandable since the $\mathrm{Li}$ ions are freer to approach the graphene plane in this case. For the on-top site attachment, the graphene plane is marginally buckled with the $\mathrm{C}$ atom that is attached to the Li atom pushed into the plane by less than $0.05 \AA$. For the hollow site, the graphene sheet remains planar.

For $100 \%$ coverage on the on-top sites, Yang considered Li adatoms alternating above and below the graphene layer [14]. After a careful analysis, Yang concluded that the $(1 \times 1)$ cell was adequate to describe this system. Yang found the counter-intuitive structure where the $C$ atoms are pushed into the plane, in direct reversal of the situation in graphane where the $\mathrm{H}$ adatoms are responsible for pulling the $C$ atoms out of the plane. Yang found strong metallic behaviour for this case involving Li on graphene with a peak in the electronic density of states at the Fermi level.

Our calculations for $100 \%$ coverage on the on-top sites reveal that the Li adatoms attaching on the same side of the graphene sheet has a lower energy compared with Li adatoms attaching alternately on opposite sides of the graphene sheet. This we find to be a surprising result, since it is different from that of $\mathrm{H}$ on graphene. Yang did not report on the case involving Li attaching on the same side of the graphene sheet.

We discovered that one needs to be very careful about the relaxation of the Li adatoms at the on-top sites on the same side of the graphene sheet. For our initial set of calculations, we considered a $(1 \times 1)$ cell. Initially, we placed the two Li adatoms at a distance of $1.80 \AA$ directly above the two $\mathrm{C}$ atoms. In this case, the $\mathrm{Li}$ adatoms relaxed symmetrically to a $\mathrm{Li}-\mathrm{C}$ bond length of $2.13 \AA$ above the graphene sheet. The graphene sheet still remained planar. The energy of this bonded system was higher than the sum of the energies of the $(1 \times 1)$ graphene cell and that of two isolated Li atoms by an amount of $3.69 \mathrm{eV}$. On this basis, it is easy to dismiss the stability of the Li adatoms attaching at the on-top sites on the same side of the graphene layer. For this configuration, the Li-Li bonds are constrained to $1.44 \AA$ which should be compared with the nearest-neighbour distance of $3.02 \AA$ for $L i$ in the BCC structure. Clearly, this is an energetically unfavoured situation which explains the high energy state of the system. Surprisingly, the atomic relaxation scheme is unable to break this symmetry as the following analysis shows.

When we apply a slight staggering to the initial positions of the Li adatoms, a very different scenario results. Here, we placed the $\mathrm{Li}$ adatoms asymmetrically at $1.88 \AA$ and $1.73 \AA$ above the respective $\mathrm{C}$ atoms. After relaxation, the $\mathrm{Li}-\mathrm{C}$ bond lengths relax to $4.43 \AA$ ("high") and $2.12 \AA$ ("low"), respectively. The energy of this 
bonded system is lower than the sum of the energies of the $(1 \times 1)$ graphene cell and of two isolated Li atoms by an amount of $2.58 \mathrm{eV}$. We refer to this energy difference as the binding energy per $(1 \times 1)$ cell. The odd result is the rather large Li-C bond length of $4.43 \AA$ for the "high" $\mathrm{Li}$ adatom. We propose that the $\mathrm{Li}-\mathrm{Li}$ interactions stabilise this arrangement. The shortest $\mathrm{Li}-\mathrm{Li}$ bond length is $2.72 \AA$ as shown in Fig. 1, which is less than the nearest-neighbour distance of $3.02 \AA$ for $\mathrm{Li}$ in the BCC structure. An analysis of the charge density distribution shows that the two Li adatoms in the calculational cell are firmly bonded to each other. The "low" Li adatom is strongly bonded to the graphene, but the "high" Li adatom is too far from the graphene sheet to be strongly bonded directly to the graphene sheet. This Li adatom is strongly held in place due to its bonding with its neighbouring Li adatoms. The co-ordination number for the "high" Li adatom is 3, and for the "low" Li adatom is 4.

The energy of this system is lower than the sum of the energy of a single Li adatom at the on-top site of the $(1 \times 1)$ graphene sheet and the energy of a single isolated Li atom by an amount of $1.64 \mathrm{eV}$ which points to the fact that this is a strongly bonded system.

We relaxed the graphene lattice constant for this system comprising the two Li adatoms at the on-top sites on the same side of the $(1 \times 1)$ graphene sheet in the staggered initial configuration. We found that the lattice expands from $2.47 \AA$ to $2.56 \AA$, with a relaxation energy of $0.15 \mathrm{eV}$. The $\mathrm{Li}-\mathrm{C}$ bond lengths remain essentially unchanged at $4.45 \AA$ and $2.12 \AA$, respectively.

For Li adatoms attached on alternate sides of the graphene layer, the Li adatoms relax symmetrically to a $\mathrm{Li}-\mathrm{C}$ bond length of $2.03 \AA$ irrespective of whether the initial positions of the two Li adatoms are symmetric or not. The binding energy per $(1 \times 1)$ cell for this system is $2.36 \mathrm{eV}$ which is less than that for the case of both the Li adatoms attaching asymmetrically on the same side of the graphene sheet by an amount of $0.22 \mathrm{eV}$.

Fig. 2 shows the density of electronic states for: (i) pure graphene $(1 \times 1)$, (ii) the two asymmetric on-top site adatoms of Li on the same side of the graphene sheet, and (iii) the two on-top site adatoms of $\mathrm{Li}$ on opposite sides of the graphene sheet. The figure indicates that there are slight changes to the densities of states for (ii) and (iii) compared to (i) far below the Fermi level, although the overall density of states for (ii) is shifted downwards in energy compared with (i) and (iii). Both (ii) and (iii) show significant changes to the density of states at the Fermi level compared with (i), with (iii) having a greater peak just below the Fermi level. The plot indicates that there are more electrons of high energy (just below the Fermi level) for (iii) compared with (ii). This gives

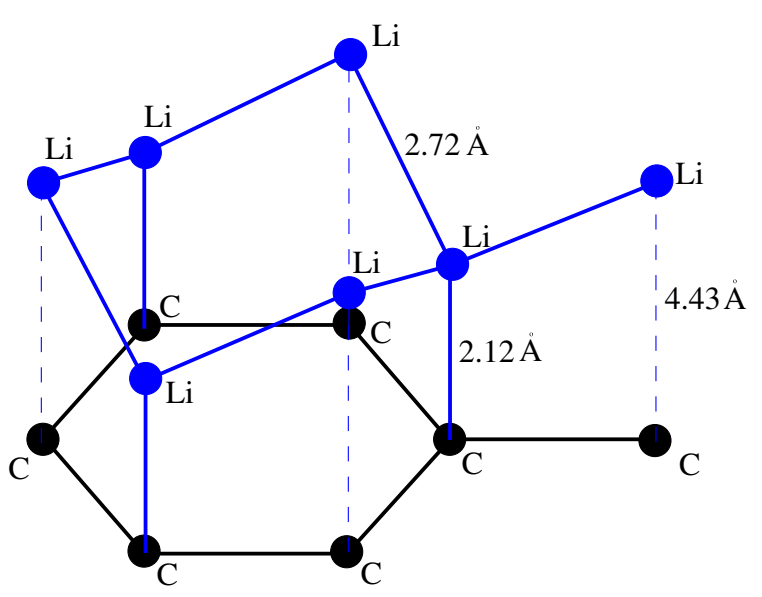

Fig. 1. The $(1 \times 1)$ cell depicting the two asymmetric on-top site adatoms of Li on the same side of the graphene sheet after relaxation. Not drawn to scale. $\mathrm{C}=$ carbon, $\mathrm{Li}=$ lithium at the "low" distance of $2.12 \AA$ above the $\mathrm{C}$, and at the "high" distance of $4.43 \AA$ above the C. The Li-Li bond is $2.72 \AA$.

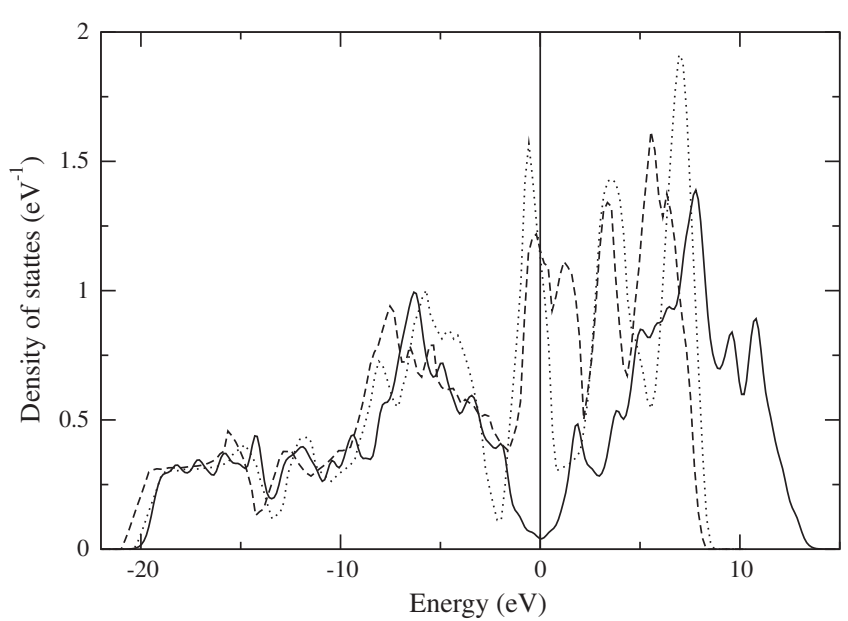

Fig. 2. The electronic density of states for: (i) pure graphene $(1 \times 1)$ (solid), (ii) the two asymmetric on-top site adatoms of $\mathrm{Li}$ on the same side of the graphene sheet (dashed), and (iii) the two on-top site adatoms of $\mathrm{Li}$ on opposite sides of the graphene sheet (dotted). The Fermi energy is set at $0 \mathrm{eV}$.

some justification for the relative stability of (ii) over (iii). We conclude that the bonding is ionic in nature with a net transfer of electronic density from the Li to the graphene substrate.

Our $(1 \times 1)$ results suggest that we should really view the overlayer not as a mono-layer, but as a bi-layer. Preliminary results for larger system sizes show that the Li overlayer is not smooth with multiple low energy states with varying heights for the Li adatoms. This is easily understood in terms of alternate Li adatoms being pushed out of the plane to accommodate the larger more optimal $\mathrm{Li}-\mathrm{Li}$ bonding. We do not further consider the $100 \%$ coverage of $\mathrm{Li}$ at the on-top sites of graphene for the larger calculational cells because this system with its high density of Li adatoms we consider to be difficult to create experimentally. We conclude that it is difficult to force $100 \%$ of the Li adatoms onto the on-top sites, which is a meta-stable site. The hollow sites are more stable. At $50 \%$ coverage the system prefers the hollow sites. For coverages beyond $50 \%$, we propose that there will be an admixture of hollow and on-top sites. It is for this reason that we have not considered any further the $(1 \times 1)$ system with $100 \%$ coverage at the on-top sites. Caragiu and Finberg [23], in their review of alkali metal absorption on graphite confirm our view that $\mathrm{Li}$ on graphene is not properly understood and that the ground state configuration is yet to be confirmed. Our submission, we believe, contributes to the understanding of the plausible structures of $\mathrm{Li}$ on graphene for the various different levels of coverages.

The hollow site is deemed to be more favourable, and this is what we consider in the next subsection.

\subsection{The $(2 \times 1)$ cell}

Here we focus on the hollow site, and we expand the calculational cell to a $(2 \times 1)$ cell to increase the number of degrees of freedom for the relaxation of the Li adatoms. Starting with symmetric initial positions for the Li adatoms at the hollow site set at $1.80 \AA$ above the graphene sheet, the system quickly relaxes to a symmetric final state with both adatoms a distance of $1.85 \AA$ above the graphene sheet, consistent with the equivalent system for the $(1 \times 1)$ cell. This we find, however, is not the lowest energy configuration for this system. The $(2 \times 1)$ cell comprising the two Li adatoms attaching on opposite sides of the graphene sheet is lower in energy compared with the two Li adatoms attaching symmetrically on the same side of the graphene sheet by an 
amount of $0.184 \mathrm{eV}$ per $(2 \times 1)$ cell. This is still not the lowest energy configuration for this system. When we begin with two Li adatoms attaching on the same side of the graphene sheet, but with slight asymmetries in their initial positions set at $1.88 \AA$ and $1.73 \AA$ above the graphene sheet, the symmetry is broken and the system is able to find an even lower energy after relaxation. The $(2 \times 1)$ cell comprising the two Li adatoms attaching asymmetrically on the same side of the graphene sheet is lower in energy compared with the two Li adatoms attaching symmetrically on the same side of the graphene sheet by an amount of $0.73 \mathrm{eV}$ per $(2 \times 1)$ cell. The asymmetry is stark with one Li adatom pulled toward the graphene layer to a distance of $2.02 \AA$ whilst the other being pushed away from the graphene layer to a distance of $3.94 \AA$. The graphene layer remains essentially planar with only slight buckling. To the best of our knowledge, this staggered configuration of Li on graphene has not been investigated before.

A careful analysis of the bond lengths in Fig. 3 shows that this system is once again stabilised by the $\mathrm{Li}-\mathrm{Li}$ interactions. There are chains of "high" Li adatoms alternating with chains of "low" $\mathrm{Li}$ adatoms. This gives rise to a corrugation potential on the surface which warrants further experimental investigation. The Li-Li bond length across the chains is $3.14 \AA$, which is slightly greater than the nearest-neighbour distance in bulk Li. The Li-Li bond length along the chains is $2.49 \AA$ which is just the distance between the hollow sites in graphene.

In Fig. 4 we plot the electronic charge density of this system to investigate the nature of the bonding of this system and the basis for the stability. The diagram indicates that this system is stabilised by the Li-Li interactions along the chains, and there is some distribution of electron density between the staggered Li adatoms. However, the overall density of electrons is low (the iso-surface is set at $0.06 \mathrm{eV} / \AA^{3}$ ). The bulk of electronic density resides in the graphene layer, which suggests transfer of electronic density from the $\mathrm{Li}$ to the graphene resulting in ionic bonding.

We spent a significant effort to investigate the extent to which this is indeed the lowest energy configuration. In particular, we investigated the possible dimerization of the Li adatoms. We considered several initial configurations of the Li adatoms attaching asymmetrically on the same side of the graphene layer at the hollow sites and with slight $x y$-displacements of the Li adatoms in the plane of the graphene layer. Our extensive search leads us to conclude that there is no tendency for dimerization, and that the $\mathrm{Li}$ adatoms prefer to remain located directly above the hollow sites in the asymmetric final state.

\subsection{Lower coverages of $\mathrm{Li}$ on graphene}

We considered $25 \%$ coverage of Li adatoms attaching on the same side of the graphene sheet at the hollow sites in the $(2 \times 2)$ cell. This constitutes rows of $\mathrm{Li}$ adatoms on the graphene

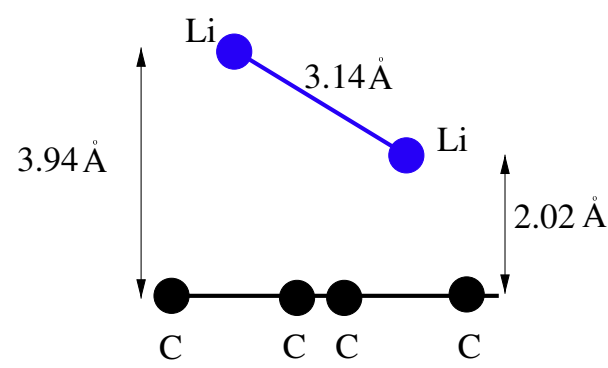

Fig. 3. The fully relaxed $(2 \times 1)$ cell in front view with the Li adatoms attaching asymmetrically on the same side at the hollow sites. Not drawn to scale. $\mathrm{C}=$ carbon, $\mathrm{Li}=$ lithium at the "low" distance of $2.02 \AA$ above the $\mathrm{C}$, and at the "high" distance of $3.94 \AA$ above the C. The Li-Li bond is $3.14 \AA$.

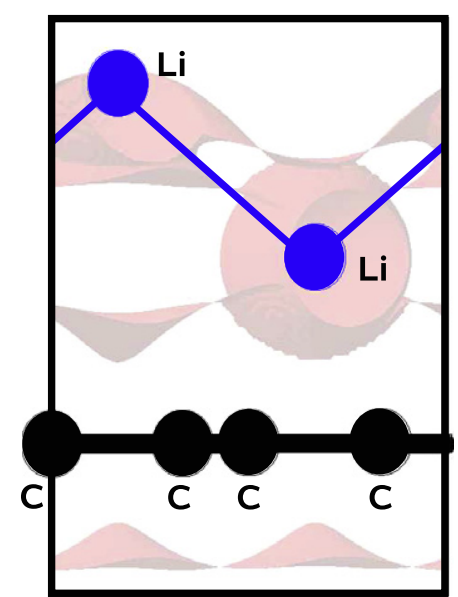

Fig. 4. The charge density plot for the fully relaxed $(2 \times 1)$ cell with the iso-surface set at $0.06 \mathrm{eV} / \AA^{3}$.

sheet alternating with valleys marked by the absence of $\mathrm{Li}$ adatoms. Because of the hexagonal symmetry of the system, there are three degenerate cases all with the same energy. Our fully relaxed calculations for this system show that along the rows the Li adatoms remain centred at the hollow sites. There is no tendency for dimerization of the $\mathrm{Li}$ adatoms, with the $\mathrm{Li}-\mathrm{Li}$ bond remaining at $2.46 \AA$ which is simply the distance between the hollow sites. Independent of the initial positions of the Li adatoms (i.e. symmetric or asymmetric initial positions, with or without $x y$-displacements in the plane of the graphene layer), the final relaxed configuration is symmetric, i.e. not staggered, with the Li adatoms a distance of $1.83 \AA$ above the graphene sheet. The binding energy for this system is $2.64 \mathrm{eV}$ per $(2 \times 2)$ cell. This system has a relatively low density of Li adatoms compared with the previous cases considered above. This enables the Li adatoms to relax readily to a symmetric final configuration.

We also considered an isolated pair of Li adatoms attached on the same side of the graphene sheet at two nearest-neighbour hollow sites. We model this system by constructing a $(5 \times 5)$ cell which is sufficiently large to minimize $\mathrm{Li}-\mathrm{Li}$ interactions between cells. We considered various initial positions of the Li adatoms, namely symmetric and asymmetric initial positions, with or without $x y$-displacements in the plane of the graphene layer. Our fully relaxed results show a configuration where the $\mathrm{Li}$ adatoms repel each other slightly from a bond length of $2.46 \AA$, which is the distance between the hollow sites, to $2.91 \AA$ which may be compared with the Li bond length of $3.02 \AA$ in the BCC structure. The Li adatoms are pushed away slightly from the hollow sites, and are lodged at a distance of $1.85 \AA$ above the graphene sheet. There is some buckling of the graphene sheet in the neighbourhood of the Li adatoms. The binding energy of this system is $2.62 \mathrm{eV}$. This repulsive interaction between the $\mathrm{Li}$ adatoms gives credence to our earlier conclusions that the Li adatoms prefer the hollow sites rather than the on-top sites - the latter forces $\mathrm{Li}$-Li interactions over shorter distances (compared with bulk $\mathrm{Li}$ ) which is energetically unfavourable. This, of course, has enormous consequences for the electronic structure and the metallic nature of this system.

\section{Conclusions}

We considered Li adatoms on graphene, and concluded that for $100 \%$ coverage at the on-top sites, the equilibrium configuration is staggered in nature with alternate Li adatoms pulled in to a distance of $2.12 \AA$ ("low") and pushed out to a distance of $4.43 \AA$ ( "high") from the graphene plane. The "high" Li adatoms 
are too far from the graphene sheet to be bonded to the graphene. This system is stabilised by the Li-Li interactions, which is verified by an analysis of the charge density distribution. Our results suggest that we should really view the overlayer not as a mono-layer, but as a bi-layer. For larger system sizes we note that the Li overlayer is not smooth since it has multiple low energy states with varying heights for the Li adatoms. This is understood in terms of alternate $\mathrm{Li}$ adatoms being pushed out of the plane to accommodate the larger more optimal $\mathrm{Li}$-Li bonding.

For $50 \%$ coverage at the hollow sites, our calculations on the $(2 \times 1)$ cell, which provides more degrees of freedom for relaxation and symmetry-breaking, show that the lowest energy configuration corresponds to Li adatoms in alternate positions being pulled into and pushed out of the graphene plane in a staggered configuration. There are chains of "high" $\mathrm{Li}$ adatoms alternating with chains of "low" $\mathrm{Li}$ adatoms. The $\mathrm{Li}$ - $\mathrm{Li}$ bond length across the chains is $3.14 \AA$, which is slightly greater than the nearest-neighbour distance in bulk $\mathrm{Li}$. The $\mathrm{Li}-\mathrm{Li}$ bond length along the chains is $2.49 \AA$ which is just the distance between the hollow sites in graphene.

The single isolated dimer shows a slight tendency for repulsion when placed at neighbouring hollow sites. This behaviour helps explain the staggered structure that we see for the more densely packed surfaces. Our results for the $(2 \times 2)$ cell at $25 \%$ coverage at the hollow sites exhibit no staggering as the Li adatoms remain at the hollow sites and relax symmetrically to a distance of $1.83 \AA$ above the graphene sheet. This constitutes rows of Li adatoms on the graphene sheet alternating with valleys marked by the absence of Li adatoms.

\section{Acknowledgments}

REM expresses gratitude to the National Institute for Theoretical Physics for financial support. N.C. wishes to thank Professors Jo- han Malherbe and Max Braun for useful discussions. The authors would like to thank Prof. Jannie Pretorius for his assistance with the use of the VASP electronic structure codes. Alexander Mavromaras has been especially helpful in assisting us with technical matters related to the use of Medea and VASP.

\section{References}

[1] K.S. Novoselov, A.K. Geim, S.V. Morozov, D. Jiang, Y. Zhang, S.V. Dubonos, I.V. Grigorieva, A.A. Firsov, Science 306 (2004) 666.

[2] A.K. Geim, K.S. Novoselov, Nat. Mater. 6 (2007) 183

[3] P.R. Wallace, Phys. Rev. 71 (1947) 622.

[4] K.S. Novoselov, A.K. Geim, S.V. Morozov, D. Jiang, Y. Zhang, M.I. Katnelson, S.V. Dubonos, I.V. Grigorieva, A.A. Firsov, Nature (London) 438 (2005) 197.

[5] Y. Zhang, Y.W. Tan, H.L. Stormer, P. Kim, Nature (London) 438 (2005) 201.

[6] A.H. Castro, F. Guinea, N.M.R. Peres, K.S. Novoselov, A.K. Geim, Mod. Phys. 81 (2009) 109.

[7] D. Lueking, H.R. Gutierrez, D.A. Fonseca, D.L. Narayanan, D.V. Essendelft, P. Jain, C.E.B. Clifford, J. Am. Chem. Soc. 128 (2006) 7758

[8] N.R. Ray, A.K. Srivastav, R. Grotzschel, In search of graphene - a twodimensional hydrocarbon, 2008. arXiv: 0802.3998v1.

[9] D.C. Elias et al., Science 323 (2009) 610. arXiv: 0810.4706v1.

[10] J.O. Sofo, A.S. Chaudhari, G.D. Barber, Phys. Rev. B 75 (2007) 153401.

[11] K.T. Chan, J.B. Neaton, M.L. Cohen, Phys. Rev. 77 (2008) 235430.

[12] M. Farjam, Rafii-Tabar, Phys. Rev. B 79 (2009) 045417.

[13] M. Khantha, N.A. Cordero, L.M. Molina, J.A. Alonso, L.A. Girifalco, Phys. Rev. B (2004) 70125422.

[14] Yang Chih-Kai, Appl. Phys. Lett. 94 (2009) 163115.

[15] G. Kresse, J. Hafner, Phys. Rev. B 47 (1993) 558.

[16] G. Kresse, J. Hafner, Phys. Rev. B 49 (1994) 14251

[17] G. Kresse, Furthmuller, Phys. Rev. B 54 (1996) 11169

[18] G. Kresse, Furthmuller, Comput. Mater. Sci. 6 (1996) 11169.

[19] J.P. Perdew, K. Burke, M. Ernzerhof, Phys. Rev. Lett. 77 (1996) 3865.

[20] P.E. Blochl, Phys. Rev. B 50 (1996) 17953.

[21] H.J. Monkhorst, J.D. Pack, Phys. Rev. B13 (1976) 5188.

[22] M.Z.S. Flores, P.A.S. Autreto, S.B. Legoas, D.S. Galvao, Nanotechnology 20 (2009) 465704.

[23] M. Caragiu, S. Finberg, J. Phys. Condens. Matter 17 (2005) R995. 